\title{
National Dementia Strategy: well intentioned - but how well founded and how well directed?
}

\author{
Ian Greaves and David Jolley
}

\begin{abstract}
The National Dementia Strategy was published in February 2009. It was eagerly anticipated and has generated a good deal of sustained media interest, widened awareness, and heightened expectation of better help for people with dementia. The three ambitions of the strategy - to raise awareness, facilitate assessment, and improve services - are unquestionably correct; but the assumptions, emphases, and economic predictions are questionable. Encouraging reliance on referral to secondary care centres, and exaggerated claims for the effectiveness of interventions to achieve clinical improvement and cost savings, may lead to disappointment and frustration. The infrastructure of care and treatment of people throughout the course of dementia will be better informed and delivered by a collaborative model which respects the knowledge and commitment contained within families and primary care. Better services will require substantial redirection of resources.
\end{abstract}

\section{Keywords}

dementia; primary health care; public policy; strategy
I Greaves, GP and honorary reader, FRCGP, Gnosall Medical Centre and Staffordshire University, Stafford. D Jolley, consultant psychiatrist and honorary reader, FRCPsych, Manchester University and Pennine Care NHS Trust, Manchester.

Address for correspondence

David Jolley, Gnosall Medical Centre and Staffordshire University, Gnosall Health Centre, Brookhouse Road, Gnosall, Stafford, ST20 0GP.

E-mail: David.jolley@manchester.ac.uk

Submitted: 13 March 2009; Editor's response: 11 May 2009; final acceptance: 24 July 2009.

OBritish Journal of General Practice 2010; 60: 193-198.

DOI: 10.3399/bjgp10X483553

\section{INTRODUCTION}

Although huge strides have been made in dementia care over a period of 40 years, the publication of a national dementia strategy is seen to be a worthwhile step towards increasing the profile of this important disorder and attracting resources to improve services. ${ }^{1,2}$ Its launch was predated by publication of detailed advice on best practice, ${ }^{7}$ and a series of studies summarising the current situation in this country and other parts of the world. $^{3-6}$

The National Dementia Strategy seeks radical change. Its commendable threefold ambition is to raise awareness of dementia (and related conditions); facilitate early investigation, diagnosis, and treatment; and improve services for people with dementia and their families. But in arguing its case, the Strategy may be too strident in its criticism of existing practices in this country, overly confident of the benefits of specialist services for all, and unrealistic in suggesting that the price of improved care will be met from cost savings.

While supporting the aims of the Strategy, we will suggest from our experience that many patients and families will be better provided with affordable assessment and continued support by strengthening the activities of primary care, rather than referral of everyone to secondary care centres. In addition, we point out that major problems arise when patients experience complex and severe symptoms. It is in these circumstances that revision of services and additional investment is most urgently needed.

\section{RAISING AWARENESS}

The UK led the world from the late 1960s in creating specialist community-orientated services for older people with mental health problems, including dementia. ${ }^{8,9}$ Yet it is declared that awareness of, and services for, dementia in England are among the poorest in the developed world. This assertion is based on a limited and oddly designed survey sponsored by Pfizer, ${ }^{10}$ and a European study which drew on marketing data of sales of cholinesterase inhibitors. ${ }^{11}$ The latter estimated that only $18 \%$ of people with Alzheimer's disease in the UK were 


\section{How this fits in}

The National Dementia Strategy was published in February 2009. The three ambitions of the strategy - to raise awareness, facilitate assessment, and improve services - are unquestionably correct; but the assumptions, emphases, and economic predictions are questionable. This article discusses the proposal to persist with a model which relies on referral of all cases to secondary care and suggests the adoption of a tiered or stepped model which is successful with other disorders. This encourages and makes best use of primary care expertise, is more cost-effective, and more acceptable to patients. competence to make decisions in the future. Families and professional carers may similarly feel helped to find an explanation for the changes and troubles they have been observing.

But the label requires that the Driver and Vehicle Licensing Agency and car insurance company are informed, with the risk that the patient's driving licence will be withdrawn or become subject to frequent review. Freedom and equality can no longer be assumed. Clinics and other organisations may require that capacity be formally checked and documented whenever decisions are being made concerning treatment or care. An industry emphasising the continued 'personhood' of people with dementia has grown, in an attempt to counter the damaging depersonalisation that labelling can produce. ${ }^{16}$

The affirmation: 'diagnosis is the gateway for care' $^{8}$ is misleading. A great deal of appropriate care and support that people require is given naturally in response to functional needs, by family, friends, and services. ${ }^{17}$ Families or individuals may feel something has been gained when the condition is named; but what does it mean and what will it mean to them? The natural history of dementia, including Alzheimer's disease, is very variable and thus differs widely from individual to individual. ${ }^{18}$

Professional psychosocial interventions can be valuable, but if given too early may exacerbate support needs or increase carer anxiety. ${ }^{12}$ For many patients, for much of the time, there will be little advantage in altering the main elements of care they were receiving before diagnosis. What is important is that psychosocial interventions can be supplemented by statutory services if and when needed. Economic arguments from the US, referenced in the Strategy, cite reduced rates of admission to care homes and reduced morbidity among carers, and thus reduced costs consequent upon early intervention. Yet they stand alongside other reports that do not confirm such benefits. ${ }^{19}$ Small and uncertain improvements in a community care programme in south-east England were achieved but at an additional cost of over $20 \%$ rather than a cost saving..$^{20}$

It is only pharmacological prescribing that requires a specific diagnosis, and then only for substances licensed to treat Alzheimer's disease. These are useful but their contribution to overall care and outcomes is limited. ${ }^{14}$

\section{EARLY INVESTIGATION, DIAGNOSIS, AND TREATMENT}

A comprehensive primary care base is one of the strengths of the NHS. Yet primary care has been repeatedly identified as the Achilles' heel of 
dementia services. It is caricatured as being slow to identify or diagnose dementia, slow to refer to secondary services, and lacking in confidence in managing the condition. ${ }^{4-6}$ The National Dementia Strategy draws from this assumption, stating that primary care should be better trained to identify people in difficulty but then should refer immediately to secondary care in the form of a 'memory service'. It is proposed that primary care should be delivering early intervention and rapid throughput (as is the approach of the Croydon Memory Service), and receiving patients back into primary care or community mental health teams for older people. ${ }^{15}$

Referral to a secondary care centre is said to be needed because diagnosis of dementia (full investigation and differential diagnosis of cognitive change) is difficult. In contrast, publications from the Guy's Hospital Community Mental Health Service for Older People, demonstrated some years ago that differential diagnosis can be achieved accurately by generic team members. ${ }^{21}$ It is important that all patients receive a comprehensive review of their general health and current treatment, and some may require additional investigations, such as brain scans. ${ }^{22}$ These are necessary to identify treatable causative or associated pathologies including depression, and to avoid false-positive diagnoses of dementia when altered memory is a benign associate of ageing. The Croydon Memory Service employs a fairly simple protocol of standard assessment schedules to make the diagnosis quickly and efficiently by generic team members.

\section{AN ALTERNATIVE APPROACH: GNOSALL MEMORY CLINIC}

An alternative to referral to expensive and stigmatised secondary mental health services is the provision of their expertise within primary care. ${ }^{23} \mathrm{We}$ at the Gnosall Memory Clinic have 3 years' experience of this approach and its effectiveness and have noted important features of a memory clinic (Box 1). Practice clinicians are encouraged to identify people in difficulty. Patients are seen by a specialist without delay and without stigma at a memory clinic in primary care. Full advantage is taken of the depth of knowledge of the individual, their family, and local resources, which is held within the practice. Potential patients are identified either at routine clinical contact or via their attendance for review on risk registers. Regular history taking and examination is supplemented by clock drawing.

The practice health visitor at Gnosall Memory Clinic has acts as the key liaison figure for the
Box 1. Essentials of the primary-care based Gnosall Memory Clinic.

- Within the patients' practice

- Patients and carers seen at health centre or at home

- Monthly sessions from specialist with availability between times

- Patients identified by clinicians at routine contact or at vascular risk register visits

- Clock drawing and BASDEC screening

- Health visitor practice lead/liaison

- Protocol of history, examination, and investigations - using the richness of knowledge of the patient, family, and local resources

- Assessment and treatment plan summarised in a letter - shared with patient and all other relevant parties

- High penetration (predicted prevalence of dementia)

- High satisfaction

- No stigma

- Cost savings

- Follow through within the practice

BASDEC $=$ Brief Assessment Schedule Depression Card.

memory clinic and completes a full history and review of circumstances in preparation for the specialist clinic at the practice. She works to an agreed protocol and can make contact with the specialist between monthly clinics by telephone or email. The assessment rate per population at risk has been sustained at three times the rate reported by the best performing secondary care clinics. The numbers approach the estimated prevalence of dementia in the practice population.

Very few patients have required referral on to specialist old age psychiatry (three patients in 3 years). Patients and carers express high satisfaction with their experience, which includes receipt of a letter that summarises findings, and further plans for investigation, treatment, and follow-up. Interestingly, systematic questioning revealed very little interest in the idea of a special support group for individuals and families with dementia; instead, a preference was expressed for meetings to address the multiple problems of frail older people. The clientele of the clinic reflects the epidemiology of dementia, with a preponderance of women over 75 years living alone or with a carer spouse.

The approach taken at the Gnosall Memory Clinic maximises the strengths of primary care in assessment and investigation and makes use of them in the ongoing support of individuals who remain recognised, respected, and not displaced 
from their natural community. Costing demonstrates considerable savings in comparison to a secondary care centre service. We suggest that this three-tiered model of memory services (primary, secondary, and tertiary), in which $90 \%$ of patients remain within the first tier, should be adopted more widely. ${ }^{24}$

A similar model is current in child and adolescent psychiatry, and recommended in the stepped approach to the care and treatment of people with depression. ${ }^{25}$ This will put dementia into proper perspective, strengthen primary care and its contributions, and make economic use of expertise and resource. Such a pioneer venture benefits from the enthusiasm and shared vision of its sponsors and participants. We believe that the refreshing collaboration between primary and secondary services, which is its essence, will be transmitted to other centres that adopt it, and that the full benefits will be realised by a whole-system approach.

\section{IMPROVING SERVICES}

There is great emphasis within the Strategy on investment in the 'front end' of services at the stage when individuals are minimally impaired. Calculations which suggest that this investment will reap financial rewards by saving expenditure on services for the more disabled and disturbed are less than convincing. The implementation and impact analysis modules of the Strategy are notable for their limitation of language and frank admission that it is not possible to provide sound cost estimates for any of the necessary investments or their outcomes. ${ }^{26,27}$

We need to do more for people who become impaired and disabled by dementia. It stretches optimism beyond reason to believe that sufficient resources can be released simply by investing in early diagnosis.

There are particular settings which demand attention and action.

- The hazards of admission to a general hospital (Objective 8 of the Strategy) are recognised to be high for everyone but more so for people with dementia. Ownership of this problem by commissioners and the management of acute trusts is essential if it is to be combated. One session of consultant psychiatrist time for every large general hospital is likely to prove cosmetic, especially if that consultant is employed by a mental health trust. What is required is correction of root-cause defects in service design and conduct in the massive 'other world' of the acute trust.

- Costs and problems are concentrated within care homes (Objective 11 of the Strategy). The Strategy urges better training and education and increased community psychiatric nurse support to the staff of homes. This is commendable, but again shies away from root causes: people who work in care homes are poorly paid. This reflects the low standing in our culture of older people with dementia and everything associated with them. There is often rapid turnover of staff. Much hands-on care is delivered by people who have not been long in this country, have limited English, and struggle to understand the special needs of older people altered by dementia. This is a huge nettle to be grasped.

- Much can be done to secure better quality of life and better economy if people can be supported in private households or very sheltered housing schemes. There are changes in the population profile which suggest more people may be supported for longer at home, with increased survival of male spouses. ${ }^{28}$ Thus, additional investment in services for individuals and carers at home is certainly to be encouraged and likely to reap cost benefits as well as quality gains.

Patients and carers plead for support that stays with them throughout the course of their dementia. This includes the very hard times when they move from one mode of care to another, and when dependency and disturbance become greater. The untried innovations of dementia advisers and peer support are proffered as possible solutions. It is not unreasonable to explore and evaluate their potential.

Additional investment in a well-educated, competent, and rewarded workforce (Objective 13), seems better suited to pay dividends. Primary care, though perhaps falling short in some ways, ${ }^{29}$ is the nearest thing to continuity that most patients have. GPs know about patients and their families and all the local sources of help, and they never discharge patients. If this is strengthened by drafting in expertise and regular reviews with specialists, then there will be an enduring safety net. Primary care needs more influence when patients stray into a general hospital or care home, and the personhood of patients needs to be better respected and preserved through the various challenges that dementia presents for the individual, their friends, and family in crises and more peaceful times, to its natural ending.

\section{IMPLEMENTATION AND IMPACT}

The publicity and media interest released by the publication of the Strategy have raised expectations. These are being felt by professionals 


\section{COMMENTARY}

The National Dementia Strategy ${ }^{1}$ highlights that dementia care and services are fragmented, poorly coordinated, and vary in quality across the UK. ${ }^{2,3}$ It is depressing to see that these issues had been identified several years ago, ${ }^{4,5}$ and also that GPs' lack of training and confidence in making a diagnosis of dementia seem, if anything, to have deteriorated since $2000{ }^{2,3,6}$ Unfortunately, the idea that making an early diagnosis of dementia could engender further fear and stigma still seems to have some currency, while current thinking and good practice both suggest that sharing the diagnosis is what people with dementia and their carers want and need. ${ }^{7}$ There is also strong evidence that, for many patients, secondary care is the best place in which to make a definitive diagnosis of dementia. ${ }^{1,3}$

The paper by Greaves and Jolle ${ }^{8}$ emphasises the role of general practice, but we should probably be cautious about over-claiming for primary care. Concerns remain about the ability of general practice to provide consistent, constant, and continuous care in some settings. Poor access, lack of continuity of care, and little or no flexibility in the service are well recognised, ${ }^{9}$ and evidence from carers and people with dementia suggest that these are important concerns. ${ }^{1}$

Local models of dementia diagnosis and care may not translate easily across the whole country, and we must be careful not to create fragmented care simply by promoting the role of general practice. An integrated strategy is needed, rather than further disparate approaches, which could lead to people with dementia and their carers and families continuing to receive poor care and services. Advocating an isolationist approach serves only to support the status quo and could deter NHS and social care delivery organisations from giving dementia the priority the Strategy recommends. ${ }^{3}$

There is a real danger that this is occurring, as highlighted recently in the media. It seems that, despite dementia being a national priority, there are few levers to make local authorities focus specifically on dementia, rather than on other health problems, such as cancer. Changes at local level are not taking place quickly enough and they lack leadership. ${ }^{3}$ We need to engage in the debate about how we can facilitate ways of bringing about large scale improvements. Without this, it is unlikely that the Strategy will be delivered at all, let alone within the 5-year time frame. We need less rhetoric and more 'joined up' collaborative multidisciplinary working between health and social care. Acting alone will do little to 'roll out' this much needed Dementia Strategy. We owe it to people with dementia, carers, and their families to act together and support this Strategy.

\section{Angela Beattie,}

BSc(Hons), MSc, PhD, RGN, CPsychol (Health), Research Associate in Health Psychology and Health Services Research, Department of Social Medicine, University of Bristol, Canynge Hall, 39 Whatley Road, Bristol, BS8 2PS. E-mail: Angela.Beattie@bristol.ac.uk

\section{Provenance}

Commissioned; not peer reviewed

\section{REFERENCES}

1. Department of Health. Living well with dementia: a National Dementia Strategy. London: HMSO, 2009.

2. National Audit Office. Improving services and support for people with dementia. London: TSO, 2007.

3 National Audit Office. Improving dementia services in England: an interim report. London: TSO, 2010.

4. Beattie A, Daker-White G, Gilliard J, Means R. How can they tell? A qualitative study of the views of younger people about their dementia and dementia care services. Health Soc Care Community 2004; 12: 359-368.

5. Beattie A, Daker-White G, Gilliard J, Means R. They don't quite fit the way we organise our services: results from a UK field study of marginalised groups in dementia care. Disabil Soc 2005; 20: 67-80.

6. Audit Commission. Forget me not: mental health services for older people. London: Audit Commission, 2000.

7. Keady, J and Williams, S. Co-constructed inquiry: a new approach to generating, disseminating and discovering knowledge in qualitative research. Quality in Ageing 2007 ; 8(2): $27-36$.

8. Greaves I, Jolley D. National Dementia Strategy: well intentioned — but how well founded and how well directed? Br J Gen Pract 2010; $10.3399 /$ bjgp10X483553.

9. Campbell J. Access to primary care: advanced ... or smart? Br J Gen Pract 2007; 57: 603-604.

DOI: 10.3399/bjgp10X483814

on the front line, who find themselves simultaneously the subject of criticism for supposed failings, while being expected to provide more for more people over longer periods, with no visible additional resource at their disposal. Sadly, this is being experienced thus far as a very topdown strategy. If we are to deliver optimal, flexible responses to the needs of patients and carers, we must devolve influence to them and the frontline professionals who work with them. This encourages pride and commitment, which facilitate better use of all resources that can be tailored to individual and local needs and strengths.

\section{CONCLUSION}

The recently published National Dementia Strategy 
is not a beginning but a point in time in the progressive improvement of services for people with dementia and their families. Much has been achieved over a period of 40 years which can be built on. Maintaining respect for non-specialist care by families and friends is essential. Strengthening the potential of primary care and including it in collaborative systems will be the best way to use our new knowledge.

\section{Linked commentary}

This article has an associated commentary: Beattie $\mathrm{A}$. $\mathrm{Br} \mathrm{J}$ Gen Pract 2010; DOI: 10.3399/bjgp10X483814.

\section{Competing interests}

The authors have stated that there are none.

\section{Acknowledgements}

Thanks particularly to Drs Claire Hilton and Susan Jolley for their constructive criticism of earlier drafts.

\section{Discuss this article}

Contribute and read comments about this article on the Discussion Forum: http://www.rcgp.org.uk/bjgp-discuss

\section{REFERENCES}

1. Department of Health. Living well with dementia: a National Dementia Strategy. London: HMSO, 2009.

2. Burns A, Robert P. The National Dementia Strategy in England. BMJ 2009; 338: b931.

3. Audit Commission. Forget-me-not. Mental health services for older people. HMSO, London: HMSO, 2000.

4. Knapp M, Prince M. Dementia UK. London: Alzheimer's Society, 2007.

5. National Audit Office. Improving services and support for people with dementia. London: HMSO, 2007.

6. Ferri CP, Prince M, Brayne C, et al, for Alzheimer's Disease International. Global prevalence of dementia: a Delphi consensus study. Lancet 2005; 366(9503): 2112-2117.

7. National Institute for Health and Clinical Excellence and Social Care Institute for Excellence. Dementia: supporting people with dementia and their carers in health and social care. National Clinical Practice Guideline number 42. London: National Institute for Health and Clinical Excellence, 2006.

http://www.nice.org.uk/guidance/index.jsp?action=byID\&o=10998 (accessed 9 Dec 2009).

8. Knapp M, Comas-Herrera A, Somani A, Banerjee S. Dementia: international comparisons. A summary report for the National Audit Office. London: PSSRU, London School of Economics, 2007.

9. Draper B, Melding P, Brodaty H. Psychogeriatric service delivery: an international perspective. Oxford: Oxford University Press, 2005.

10. Bond J, Stave C, Sganga A, et al. Inequalities in dementia care across Europe: key finding of the facing Dementia survey. Int J Clin Pract 2005; 59(suppl 146): 8-14.

11. Waldemar G, Phung KT, Burns A, et al. Access to diagnostic evaluation and treatment for dementia in Europe. Int J Geriatr Psychiatry 2007; 22(1): 47-54

12. Moniz-Cook E, Manthorpe J. Early psychosocial interventions in dementia. London: Jessica Kingsley, 2009.

13. National Institute for Health and Clinical Excellence. Alzheimer's disease - donepezil, galantamine, rivastigmine (review) and memantine. Technology Appraisal TA111. London: National Institute for Health and Clinical Excellence, 2007.

http://www.nice.org.uk/TA111 (accessed 9 Dec 2009).

14. Lyle S, Grizzell M, Willmott S, et al. Treatment of a whole population sample of Alzheimer's disease with donepezil over a four year period: lessons learned. Dement Geriatr Cogn Disord 2008; 25(3): 226-231.

15. Banerjee S, Willis R, Matthews D, et al. Improving the quality of care for mild to moderate dementia: an evaluation of the Croydon Memory Service Model. Int J Geriatr Psychiatry 2007; 22(8): $782-788$.

16. Kitwood T. Dementia reconsidered: the person comes first. Maidenhead: Open University Press, 1997.

17. Eagles J, Beattie J, Blackwood G, et al. The mental health of elderly couples. I. The effects of a cognitively impaired spouse. $\mathrm{Br} \mathrm{J}$ Psychiatry 1987; 150: 299-303.

18. Holmes $\mathrm{C}$, Lovestone $\mathrm{S}$. Long-term cognitive and functional decline in late onset Alzheimer's disease: therapeutic implications. Age Ageing 2003; 32(2): 200-204.

19. Gaugler J, Kane R, Kane R, Newcomer R. Early community-based service utilisation and its effects on institutionalisation in dementia care-giving. Gerontologist 2005; 45(2): 177-185.

20. Challis D, von Abendorff R, Brown M, et al. Case management, dementia care and specialist mental health services: an evaluation. Int J Geriatr Psychiatry 2002; 17(4): 315-325.

21. Collighan G, Macdonald A, Herzberg J, et al. An evaluation of the multidisciplinary approach to psychiatric diagnosis in elderly people. BMJ 1993; 306(6881): 821-824.

22. Royal College of Psychiatrists. Forgetful but not forgotten. Council Report no. 119. London: Royal College of Psychiatrists, 2005.

23. Greening L, Greaves I, Greaves N, Jolley D. Positive thinking on memory problems and dementia in primary care: The Gnosall Memory Clinic. Community Pract 2009; 82(5): 20-23.

24. Jolley D, Greaves I, Greaves N, Greening L. Three tiers for a comprehansive regional memory service. dementia. J Dementia Care 2010; 18(1): 26-29.

25. Chew-Graham C, Baldwin R, Lovell K. Management of late-life depression. In: Chew-Graham C, Baldwin R, Burns A (eds). Integrated management of depression in the elderly. Cambridge: Cambridge University Press, 2009; 17-32.

26. Department of Health. Living well with dementia: implementation plan. London: Crown Copyright, 2009. http://www.dh.gov.uk/en/Publicationsandstatistics/Publications/Pub licationsPolicyAndGuidance/DH_094058 (accessed 9 Dec 2009)

27. Department of Health. Impact assessment of National Dementia Strategy.

http://www.dh.gov.uk/prod_consum_dh/groups/dh_digitalassets/do cuments/digitalasset/dh_094055.pdf (accessed 9 Dec 2009).

28. Dunnell K. Ageing and mortality in the UK. Popul Trends 2008; (134): 6-23.

29. Campbell J. Access to primary care: advanced ... or smart? Br J Gen Pract 2007; 57(541): 603-604. 